\title{
Non-Newtonian Thermosensitive Nanofluid Based on Carbon Dots Functionalized with Ionic Liquids
}

\author{
Helena M. R. Gonçalves, * Rui F. P. Pereira, Emmanuel Lepleux, Louis Pacheco, \\ Artur J. M. Valente, Abel J. Duarte, and Verónica de Zea Bermudez*
}

Non-Newtonian nanofluids present outstanding features in terms of energy transfer and conductivity with high application in numerous areas. In this work, non-Newtonian nanofluids based on carbon dots (Cdots) functionalized with ionic liquids (ILs) are developed. The nanofluids are produced using a simple, single-step method where the raw materials for the Cdots synthesis are glucose and waste biomass (chitin from crab shells). The use of ILs as both reaction media and functionalization molecules allows for the development of a new class of nanofluids, where the ILs on the Cdots surface represent the base-fluid. Here, the well-known benign IL 1-butyl-3-methylimidazolium chloride ([Bmim]Cl) and a novel home-made IL (1-tosylate-3-methylimidazolium triflate) [Tmi][Trif] are used. The nanofluids obtained from both substrates show, apart from high conductivity and viscosity, light absorption, and good wettability, an appealing thermal sensitivity behavior. This thermal sensitivity is preserved even when applied as thin films on glass slides and can be boosted using the surface plasmon resonance effect. The results reported demonstrate that the new Cdots/IL-based nanofluids constitute a versatile and cost-effective route for achieving high-performance thermosensitive non-Newtonian sustainable nanofluids with tremendous potential for the energy coatings sector and heat transfer film systems.

\section{Introduction}

Non-Newtonian nanofluids, or fluid suspensions of nanoparticles (NPs), represent a fairly new, interdisciplinary, and rapid evolving area with high academic relevance and extraordinary input in industry and ultimately society. ${ }^{[1]}$ As their performance largely surpasses that of currently employed heat transfer liquids, they hold great promises for applications in thermal management. For instance, the use of nanofluid-coated surfaces allows to significantly increase the energy conversion/ heat transfer, particularly between solids and liquids/gases. ${ }^{[2]}$ The large surface-tovolume ratio of NPs and the high particle mobility explain the outstanding thermal properties of nanofluids: an anomalously large thermal conductivity with respect to traditional liquids lacking NPs or incorporating larger particles, ${ }^{[3]}$ a strong temperature dependence of thermal conductivity, and a significant increase in critical heat flux in boiling heat transfer. Because of

Dr. H. M. R. Gonçalves, Prof. A. J. Duarte

REQUIMTE

Instituto Superior de Engenharia do Porto

Porto 4200-072, Portugal

E-mail: helenardrgs@gmail.com

Dr. H. M. R. Gonçalves, Prof. V. de Zea Bermudez

Chemistry Department and CQ-VR

University of Trás-os-Montes e Alto Douro

Vila Real 5001-801, Portugal

E-mail: vbermude@utad.pt

Dr. R. F. P. Pereira

Chemistry Department and Chemistry Centre

University of Minho

Braga 4710-057, Portugal

Dr. E. Lepleux, L. Pacheco

CS Instruments (CSI-AFM)

Les Ulis 91940, France

Prof. A. J. M. Valente

CQC

Chemistry Department

University of Coimbra

Coimbra 3004-535, Portugal

The ORCID identification number(s) for the author(s) of this article can be found under https://doi.org/10.1002/smll.201907661.

DOI: 10.1002/smll.201907661 their appealing and unique features nanofluids lend themselves to applications in a plethora of nano-engineering sectors ranging from space exploration, automotive industry, and energy storage, to medicine, including cancer therapy. ${ }^{[4]}$

Recently we introduced a novel nanofluid concept which relies on the functionalization of glucose-derived carbon dots (Cdots) with the commercially available ionic liquid (IL) 1-butyl-3-methylimidazolium chloride ([Bmim]Cl). The nanofluid synthesized exhibits self-improving ionic conductivity, thermotropy around $30-40{ }^{\circ} \mathrm{C}$, absorption in the ultraviolet (UV) region and emission in the visible (vis) region. ${ }^{[5]}$ The Cdots/[Bmim]Cl nanofluid was employed in a thermotropic device (TTD) with surface plasmon resonance effect (SPRE)boosted performance, and in an integrated thermo-electrooptical device, both enabling modulation of solar light (vis spectral region) and heat (near infrared spectral region). The integrated device operated, either in TT mode, or in electrochromic (EC) mode, the latter offering bright hot and dark cold modes, impressive memory effect, excellent cycling stability, and colossal coloration efficiency. All these attributes render this nanofluid potentially interest for smart window technology, allowing to envisage applications in energy-efficient buildings. ${ }^{[5]}$ 

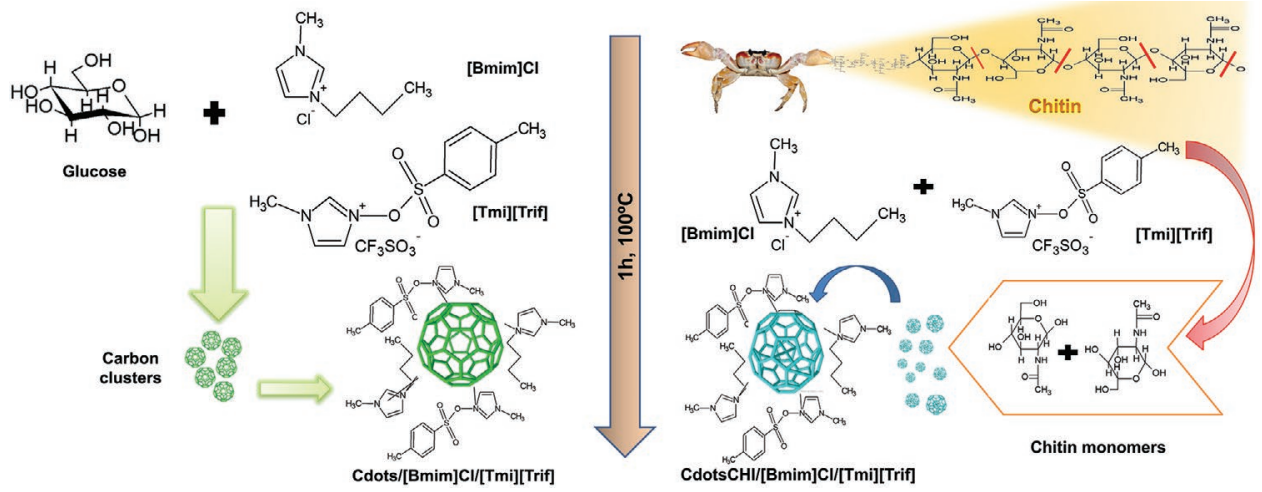

Figure 1. Scheme of the synthesis of the Cdots/[Bmim] Cl/[Tmi][Trif] and $\mathrm{Cdots} \mathrm{CHI} /[\mathrm{Bmim}] \mathrm{Cl} /[$ Tmi] $]$ Trif] nanofluids.

The rational for the combination of Cdots with $[\mathrm{Bmim}] \mathrm{Cl}$ is obvious: 1) Cdots are exciting nanometer sized, zero-dimension fluorescent carbon-based NPs. Although these nanomaterials have been proposed for applications in numerous areas, especially biosensing/bioimaging, ${ }^{[6]}$ over these past few years the perspective of using Cdots in energy applications has grown rapidly. $\left.{ }_{[}\right]$Their most attractive characteristics, in particular their inherent non-toxicity and the recent advances performed in the synthesis procedures, have made them quite attractive candidates for hot areas of research and industry. ${ }^{[8]}$ Ideally any compound exhibiting a carbon-based structure could potentially be used for the Cdots production. For this reason, the use of the widely available carbohydrates that can be obtained from waste biomass (e.g., glucose from many fruits and vegetables that are not appropriate for consumption) represents a valuable step forward to a better future; 2) [Bmim] Cl is an extensively employed, commercially available and non-toxic IL, which displays reversible thermotropy around $30{ }^{\circ} \mathrm{C}$, and strong hydrogen bond accepting ability.

Given the dual role played by $[\mathrm{Bmim}] \mathrm{Cl}$ on the synthesis and properties of the Cdots/[Bmim]Cl nanofluid ${ }^{[5]}$ (as reaction medium and functionalization molecule) and taking into account that the nature of the carbon-based precursor material employed to produce the Cdots deeply affects their physical-chemical properties, it seemed of the utmost interest to develop new strategies in terms of the IL component and of the raw carbon source, to further enlarge the potential of the Cdots/IL nanofluid platform. We sought in particular: 1) to compare the nanofluids characteristics using different raw materials and different ILs; 2) to further improve the ionic conductivity $\left(0.46\right.$ and $2.8 \mathrm{mS} \mathrm{cm}{ }^{-1}$ at 24 and $58{ }^{\circ} \mathrm{C}$, respectively, for Cdots/[Bmim $\left.] \mathrm{Cl}^{[5]}\right)$ and 3 ) to further enhance the plasmonic-induced TT effect (transmittance variation $[\Delta T]$ of $47 \% / 31 \%$ at $550 / 1000 \mathrm{~nm}$ for Cdots/[Bmim] $\left.\mathrm{Cl}^{[5]}\right)$. Keeping these goals in mind, in the present work we synthesized two novel nanofluids based on Cdots produced from chitin extracted from crab shells and glucose. In addition, the Cdots were functionalized with $[\mathrm{Bmim}] \mathrm{Cl}$ and the homemade 1-tosylate-3-methyl-imidazolium triflate ([Tmi][Trif]) IL (Figure 1). To the best of our knowledge, the latter IL has never been reported previously. This new imidazolium-based IL deliberately contains a sulfonate $\left(-\mathrm{SO}_{3}{ }^{-}\right)$group (Table $\mathrm{S} 1$ and Figure S1, Supporting Information) for two reasons: 1) it endows materials with high proton conductivity; ${ }^{[9,10]}$ and 2) it facilitates the rupture of the glycosidic bonds present in complex carbohydrates. ${ }^{[11]}$ The chitin derived nanofluid has been represented by the notation CdotsCHI/[Bmim]Cl/[Tmi][Trif]. For comparison purposes an analogue nanofluid was obtained from anhydrous synthetic glucose (Cdots/[Bmim]Cl/[Tmi] [Trif]). The wettability, viscosity, ionic conductivity, thermotropy, and optical features of both nanofluids have been thoroughly characterized and compared with those of the parent Cdots/ [Bmim] Cl nanofluid. The performance of a SPRE-induced TT device has been studied.

\section{Results and Discussion}

\subsection{Nanofluids Synthesis}

Traditional hydrothermal methods for Cdots synthesis require temperatures $\geq 200{ }^{\circ} \mathrm{C}$, reaction time $>3 \mathrm{~h}$ and/or high pressures, regardless of the raw material. ${ }^{[12,13]}$ In the presence of $[\mathrm{Bmim}] \mathrm{Cl}$, Cdots were produced from glucose after just $1 \mathrm{~h}$, at $100{ }^{\circ} \mathrm{C}$, using simple laboratory apparatus. ${ }^{[5]}$ We proposed that $[\mathrm{Bmim}] \mathrm{Cl}$ presumably participated first in the cascade reactions that led to the formation of the nucleation clusters, and once the Cdots began to grow, the $[\mathrm{Bmim}] \mathrm{Cl}$ molecules bonded to their surface, stabilizing them, ${ }^{[14,15]}$ and ultimately yielding the nanofluid.

Preliminary tests showed that if the synthesis of the Cdots/ $[\mathrm{Bmim}] \mathrm{Cl}$ nanofluid was performed with chitin instead of glucose, the reaction did not produced nanoparticles. This finding clearly indicated that $[\mathrm{Bmim}] \mathrm{Cl}$ was definitely not able to break the glycosidic bonds of chitin, probably because the latter polysaccharide, which contains acetamide groups apart from all the other functional groups present in glucose, is structurally more complex. This prompted us to design [Tmi][Trif].

The formation of the Cdots was followed in time using fluorescence intensity measurements (Figure S2). Initially there was no fluorescence observed, as the nanoparticles began to developed there was a gradual increase in fluorescence intensity. As the reaction was left to advance the fluorescence intensity reaches a maximum and then decreases, probably due to the formation of aggregates that quenches the fluorescence. At $1 \mathrm{~h}$ reaction time the reaction of glucose in the presence of both $[$ Bmim]Cl and [Tmi][Trif] reached a maximum fluorescence 
intensity upon $1 \mathrm{~h}$ (Figure S2 and Figure 1a). When glucose was replaced by chitin the CdotsCHI/[Bmim]Cl/[Tmi][Trif] nanofluid was successfully produced under the same experimental conditions in the same period of time (Figure 1b). Unlike Cdots/[Bmim]Cl and Cdots/[Bmim]Cl/[Tmi][Trif], which were produced as dark brown nanofluids, CdotsCHI/[Bmim]Cl/[Tmi] [Trif] exhibits an orange color.

\subsection{Nanofluids Surface Characterization}

The co-existence of [Bmim] $\mathrm{Cl}$ and [Tmi][Trif] during the synthesis of the two nanofluids, plus the different origin of the Cdots, had a major impact on the global physical-chemical properties of the resulting nanofluids.

The elemental analysis data represented in the bottom inset of Figure 2a and in the left inset of Figure $2 \mathrm{~b}$ reveal the presence of sulfur atoms in Cdots/[Bmim]Cl/[Tmi][Trif] and CdotsCHI/[Bmim]Cl/[Tmi][Trif], respectively, providing support to the Cdots functionalization with [Tmi][Trif].

Figure 2a allows inferring that the NPs of Cdots/[Bmim] $\mathrm{Cl} /[$ Tmi][Trif] have spherical morphology. The dynamic light scattering (DLS) data (Figure S3, Supporting Information) indicated the occurrence of a single population of NPs homogeneously distributed with an average size of $44 \mathrm{~nm}$. This means that the Cdots in this nanofluid are considerably bigger $\left(\approx 6.5\right.$ times) than those found in Cdots/[Bmim] $\mathrm{Cl}^{\left[{ }^{[5]}\right.}$ On the other hand, the high-resolution transmission electron microscopy a

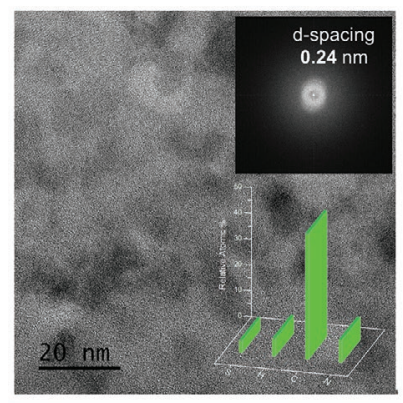

b

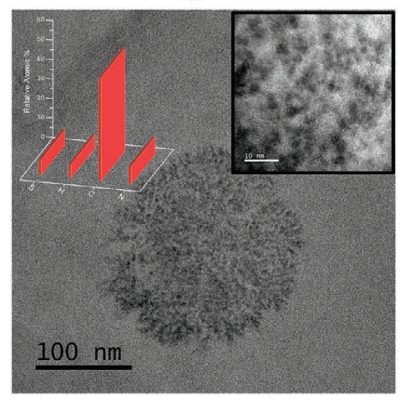

c

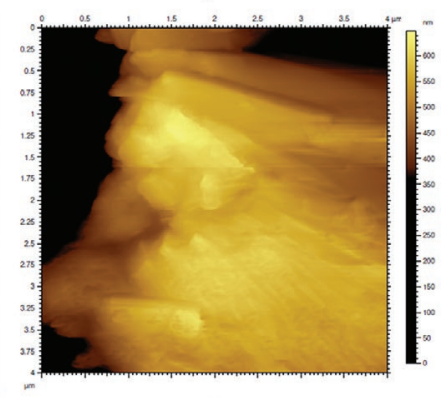

d

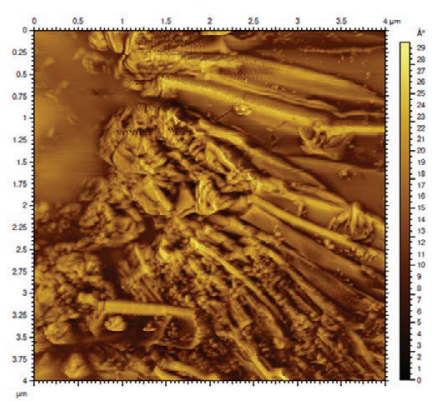

Figure 2. Structure and composition. HR-TEM images of a) Cdots/ [Bmim] Cl/[Tmi][Trif] (Top inset: FFT of the HR-TEM image; bottom inset: Elemental analysis data) and b) Cdots $\mathrm{CHI} /[\mathrm{Bmim}] \mathrm{Cl} /[$ Tmi] [Trif] (Left inset: Elemental analysis data; Right inset: HR-TEM image of CdotsCHI/ $[\mathrm{Bmim}] \mathrm{Cl} /[\mathrm{Tmi}][$ Trif] for a reaction time of $2 \mathrm{~h}$ ). AFM 2D c) topographical and d) phase images of $\mathrm{Cdots} \mathrm{CHI} /[\mathrm{Bmim}] \mathrm{Cl} /[\mathrm{Tmi}][$ Trif] obtained in resonant mode for a reaction time of $1 \mathrm{~h}$.
(HR-TEM) analysis revealed that there are larger particles, around $40 \mathrm{~nm}$, but they seem to be aggregates. Moreover, the nanoparticles medium size distribution (Figure S4) indicated a population of $13 \pm 3 \mathrm{~nm}$. These discrepancies between the data obtained by the two techniques are probably correlated with the Cdots tendency to form aggregates in water. Indeed, even though the nanofluids were passed through a $200 \mathrm{~nm}$ filter and then polydispersed using ultrason for $5 \mathrm{~min}$ before the DLS measurement, the aggregation tendency led to the DLS result. The fast-Fourier transformation (FFT) of the HR-TEM image (top inset of Figure 2a) revealed the typical lattice spacing of approximately $0.24 \mathrm{~nm}$, consistent with the (020) lattice plane of graphite. ${ }^{[16]}$ On the basis of the information obtained from DLS along with the quantities of reagents employed in the Cdots synthesis, we calculated that in their surface there must be around $2.74 \times 10^{6}$ molecules of $[\mathrm{Bmim}] \mathrm{Cl}$ and $1.96 \times 10^{7}$ molecules of [Tmi][Trif]. This allowed us deducing that the surface charge density was approximately $4.51 \times 10^{16} \mathrm{~cm}^{-2}$. We recall that in the case of Cdots/[Bmim]Cl, only 37 [Bmim] $\mathrm{Cl}$ molecules were found at the surface of the considerably smaller Cdots $(6.8 \mathrm{~nm})$, the surface charge density being $\approx 1.10 \times 10^{15} \mathrm{~cm}^{-2}$.[5]

The CdotsCHI/[Bmim]Cl/[Tmi][Trif] nanofluid presents, in contrast, according to the DLS data two distinct size populations with average sizes of 45 and $170 \mathrm{~nm}$ (Figure S3b). On the other hand, and as previously seen for Cdots/[Bmim]Cl/[Tmi] [Trif], the HR-TEM analysis revealed that the nanofluid presents a medium size population of $4.8 \pm 0.6 \mathrm{~nm}$ (Figure S4b), despite the fact that it was also possible to see micelle-like structures (Figure $2 \mathrm{~b}$ ) that could account for the two populations found in DLS. The micelle-like structures observed in the HR-TEM image (Figure $2 \mathrm{~b}$ ) could account for the two populations found. However, as these NPs were suspended in ethanol and water prior to performing the HR-TEM and DLS measurements, respectively, it was necessary to get additional evidences that these micellar structures did exist in the nanofluidic state. With this purpose, we performed AFM analysis of the CdotsCHI/ [Bmim]Cl/[Tmi][Trif] nanofluid which beautifully revealed the same micellar-like structures (Figure 2c,d). This proves that the Cdots derived from chitin and functionalized with both ILs can be considered as individual NPs with great tendency to assemble into $170 \mathrm{~nm}$-diameter micelle-like structures. To check whether the reaction time had been insufficient when the complex chitin carbohydrate was used instead of glucose, we performed again the synthesis of the CdotsCHI/[Bmim]Cl/[Tmi] [Trif] nanofluid allowing this time the reaction to evolve for $2 \mathrm{~h}$. The HR-TEM images acquired revealed a medium size population of $2.8 \pm 0.6 \mathrm{~nm}$ (Figure S4c) and afterward a the typical lattice spacing of approximately $0.24 \mathrm{~nm}$, consistent with the (020) lattice plane of graphite, ${ }^{[16]}$ and a single population of NPs with average size of $45 \mathrm{~nm}$ (Figure $2 \mathrm{~b}$ [inset]). These findings allow us stating that in the case of Cdots produced from chitin, the size heterogeneity is unequivocally related with the reaction time, corroborating previous reports that pointed out the great influence that the carbon raw material has on NPs, particularly their molecular weight. ${ }^{[16-18]}$ The calculated number of [Bmim] $\mathrm{Cl}$ and [Tmi][Trif] molecules on the chitin-derived Cdots surface was $4.39 \times 10^{6}$ and $3.15 \times 10^{7}$, respectively, for the $45 \mathrm{~nm}$ population, and $4.82 \times 10^{7}$ and $3.46 \times 10^{8}$, respectively, for the $170 \mathrm{~nm}$ 
a

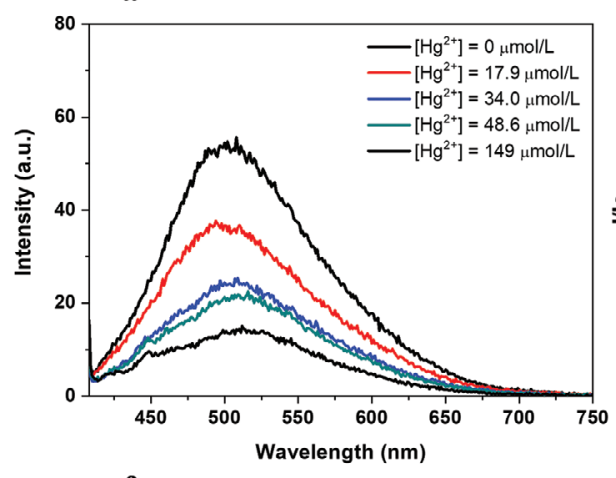

C

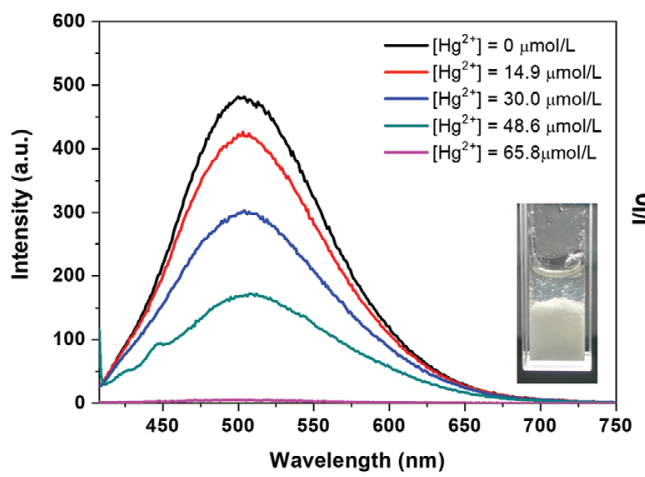

b

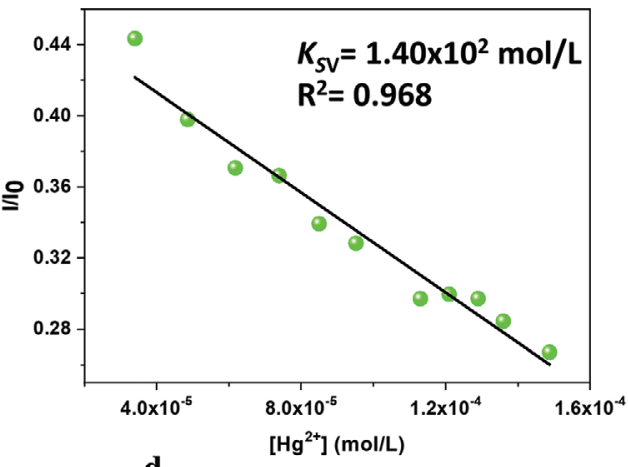

d

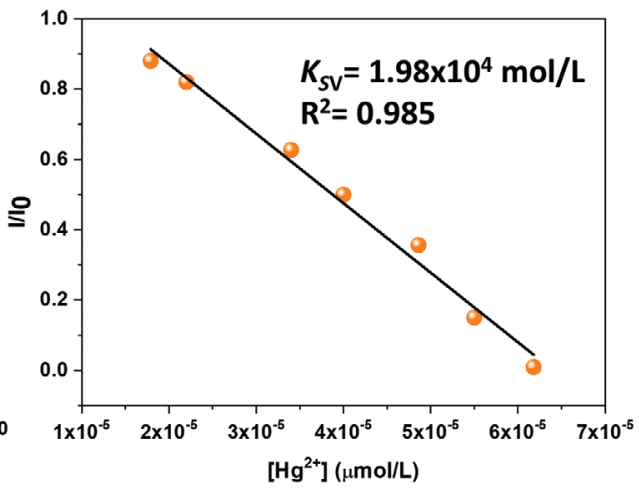

Figure 3. Fluorescence quenching. Profile for a) Cdots/[Bmim] $\mathrm{Cl} /[$ Tmi $][$ Trif $]$ and b) Cdots/CHI[Bmim] Cl/[Tmi][Trif]. Stern-Volmer plots of c) Cdots/ $[$ Bmim] $\mathrm{Cl} /[$ Tmi $][$ Trif $]$ and d) Cdots/CHI[Bmim]Cl/[Tmi] [Trif].

population. From these values we estimated a surface charge density of $6.90 \times 10^{16}$ and $7.58 \times 10^{17} \mathrm{~cm}^{-2}$, respectively.

From TG and corresponding DTG analysis (Figure S5) it can be found that [Tmi][Trif] shows two maximum degradation temperatures $\left(T_{\max }\right)$ at 270 and $391{ }^{\circ} \mathrm{C}$. The first $T_{\max }$ is due to the tosylate group whilst the second degradation step is related with the imidazolium group. ${ }^{[19,20]}$ For the Cdots/[Bmim] $\mathrm{Cl} /\left[\right.$ Tmi][Trif] nanofluid, it can been seen that two $T_{\max }$ are also observed at 270 and $406{ }^{\circ} \mathrm{C}$; the former temperature overlaps with the maximum degradation temperature of glucose (around $311^{\circ} \mathrm{C}$ ) and $[\mathrm{Bmim}] \mathrm{Cl}$ (ca. $284^{\circ} \mathrm{C}^{[20]}$ ) as suggested by an higher weight loss percentage (76\%), when compared with that for [Tmi][Trif] (around 50\%); it can also be seen a slight degradation step (green zone in Figure $\mathrm{S} 5 \mathrm{~b}$ ) with a $T_{\max }=189{ }^{\circ} \mathrm{C}$ which might be assigned to glucose degradation. The degradation of CdotsCHI/[Bmim]Cl/[Tmi][Trif] shows a 3 well defined $T_{\max }$ at 280,371 and $421{ }^{\circ} \mathrm{C}$; the latter can be assigned to the chitin degradation, ${ }^{[21]}$ whilst the first two degradation temperatures suggest that the presence of chitin induces a stabilization of the tosylate group and an opposte effect on the imidazolium groups. These results provide additional proofs of the functionalization of the Cdots surface and of the high thermal stability provided by the ILs, in particular for the chitincontaining nanofluid. These results provide additional proofs of the functionalization of the Cdots surface and of the high thermal stability provided by the ILs, in particular for the chitincontaining nanofluid.

To get insight into the organization and relative orientation of the two ILs surrounding the NPs in Cdots/[Bmim] $\mathrm{Cl} /[$ Tmi][Trif] and CdotsCHI/[Bmim]Cl/[Tmi][Trif], a simple fluorescence quenching assay was performed. Both nanofluids were exposed to increasing concentrations of mercury (II) sulfate $\left(\mathrm{HgSO}_{4}\right)$ and the fluorescence signal was recorded.

Figure 3a reveals that there was a marked decrease in the fluorescence intensity of Cdots/[Bmim]Cl/[Tmi][Trif] as the $\mathrm{Hg}^{2+}$ concentration was increased up to $149 \mu \mathrm{mol} \mathrm{L} \mathrm{L}^{-1}$. To check if this fluorescence quenching profile was due to the chemical interaction between $\mathrm{Hg}^{2+}$ and the sulfonate moiety of [ $\mathrm{Tmi}^{+}$], a similar test was performed with Cdots/[Bmim]Cl. In the latter case the fluorescence intensity did not change regardless of the $\mathrm{Hg}^{2+}$ concentration and no precipitate was formed (not shown). These results represent solid evidences that in the Cdots/[Bmim]Cl/[Tmi][Trif] nanofluid the sulfonate moiety of [Tmi][Trif] does not interact directly with the Cdots surface, being thereby oriented outward of the Cdots core and available for strong interaction with $\mathrm{Hg}^{2+} \cdot{ }^{20]}$ Moreover, as no precipitate was formed either with Cdots/[Bmim]Cl/[Tmi][Trif] and Cdots/ [Bmim $] \mathrm{Cl}$, we are led to conclude that the imidazolium cations of both ILs (i.e., $\left[\mathrm{Bmim}^{+}\right]$and $\left[\mathrm{Tmi}^{+}\right]$) remain close to the surface of the Cdots, probably interacting with carboxylate and hydroxyl groups via the $\mathrm{N}$ atoms. Based on the data extracted by the quenching profile of Cdots/[Bmim]Cl/[Tmi][Trif] it was possible to obtain an approximate Stern-Volmer constant $\left(K_{\mathrm{SV}}\right)$ for the $\mathrm{Hg}(\mathrm{II}) /$ nanofluid interaction of $1.40 \times 10^{2} \mathrm{~mol} . \mathrm{L}^{-1}$ (Figure $3 \mathrm{~b}$ ).

The results of the application of the same assay with CdotsCHI/[Bmim]Cl/[Tmi][Trif] solution also provided very useful information. Indeed when $\mathrm{Hg}^{2+}$ was added, a white precipitate was formed (inset of Figure 3c), consistent with the formation of an $\mathrm{N}-\mathrm{Hg}$ complex, ${ }^{[20]}$ and pointing out that the interaction of $\left[\mathrm{Bmim}^{+}\right]$and $\left[\mathrm{Tmi}^{+}\right]$with the Cdots surface in 
CdotsCHI/[Bmim]Cl/[Tmi][Trif] is different from that found with the other two Cdots described above. Indeed the acetoamino groups present in the chitin monomers are also likely present in the CdotsCHI/[Bmim]Cl/[Tmi][Trif] surface. Therefore in this nanofluid both imidazolium cations are, most probably, interacting with the Cdots surface through the alkyl chains, leaving the $\mathrm{N}$ atoms available for the formation of the $\mathrm{Hg}^{2+}$ complex. In this case it was also possible to obtain a typical quenching Stern-Volmer plot for $\mathrm{Hg}^{2+}$ using the following equation: $\frac{I_{0}}{I}=1+K_{\mathrm{sv}}[\mathrm{Hg}(\mathrm{II})]$, where $I_{0}$ and $I$ correspond to the fluorescence intensity without and with the metal, respectively. ${ }^{[20]}$ In the light of this approach it was possible to obtain the approximate value of $K_{\mathrm{SV}}\left(1.96 \times 10^{4} \mathrm{~mol} . \mathrm{L}^{-1}\right)$ Figure $3 \mathrm{~d}$ for the $\mathrm{Hg}^{2+}$ nanofluid interaction. When, upon each addition of $\mathrm{HgSO}_{4}$, we extracted the white precipitate and measured the fluorescence of the remaining solution (Figure 3c). In contrast with the situation observed in Cdots/[Bmim]Cl/[Tmi][Trif], in the case of CdotsCHI/[Bmim]Cl/[Tmi][Trif] it was only possible to reach a $\mathrm{Hg}^{2+}$ concentration of $48.6 \mu \mathrm{mol} \mathrm{L} \mathrm{L}^{-1}$ before total extinction of the fluorescence intensity occurred, which is about $67 \%$ less than that used for Cdots/[Bmim]Cl/[Tmi][Trif]. In conclusion, these data demonstrate that the fluorescence mechanism in the presence of $\mathrm{Hg}^{2+}$ was extremely relevant for the understanding of the relative position and the chemical interaction between the ILs and the Cdots surface.

The knowledge of the surface tension and wettability of nanofluids is fundamental, dictating their potential applications in heat exchangers and energy systems. ${ }^{[21]}$ Both properties depend on the nature, shape, size, and content of the NPs. The surface tension can be used as an indicator of NP dispersion and stability. Surface tension and static contact angle $(\theta)$ measurements are useful tools to monitor modifications at the surface of NPs. ${ }^{[21]}$ The Cdots/[Bmim]Cl/[Tmi][Trif] and Cdots/CHI[Bmim] $\mathrm{Cl} /[$ Tmi] [Trif] nanofluids are less hydrophilic (Figure S6, Supporting Information) $\left(\theta=42.03 \pm 0.15^{\circ}\right.$ and $49.82 \pm 1.97^{\circ}$, respectively) than the corresponding concentrated $95 \% \mathrm{w} / \mathrm{w}$ aqueous solutions $\left(\theta=18.11 \pm 0.42^{\circ}\right.$ and $19.9 \pm 1.06^{\circ}$, respectively), in agreement with previous studies performed with other types of NPs, ${ }^{[22]}$ and also higher than the Cdots/[Bmim $] \mathrm{Cl}$ nanofluid ${ }^{[5]}\left(\theta=27.20 \pm 0.78^{\circ}\right)$. Naik and Vinod ${ }^{[23]}$ associated the enhancement of the $\theta$ values (i.e., the decrease of hydrophilic behavior) in sulfur-based nanofluids with respect to the base fluids with extensive hydrogen bonding. The crucial role played by the sulfonate functionality is also manifested in the $\theta$ value of the $95 \% \mathrm{w} / \mathrm{w}$ aqueous solution of Cdots/[Bmim] Cl, which lacks [Tmi][Trif], and is practically identical $\left(\theta=25.03 \pm 0.83^{\circ}\right)$ to that measured for the bulk state. The slight less hydrophilic behavior of $\mathrm{CdotsCHI} /[\mathrm{Bmim}] \mathrm{Cl} /[\mathrm{Tmi}][$ Trif $]$ with respect to Cdots/[Bmim]Cl/[Tmi][Trif] is tentatively attributed to the different size of the Cdots in each nanofluid.

The stability of the nanofluid in suspension was assessed by means of zeta potential analysis. This technique is particularly relevant, providing rich information concerning the cumulative surface charge density of the NPs regardless of the number of charge ligands. At $16^{\circ} \mathrm{C}$ the zeta potential of Cdots/[Bmim]Cl/[Tmi][Trif] was $-12 \mathrm{mV}$ (Figure 4a), thus slightly higher than that reported for Cdots/[Bmim] Cl $\left(-16 \mathrm{mV}{ }^{[5]}\right)$. This was an expected result, considering the smaller size of the NPs in the latter nanofluid. The negative value found for Cdots/[Bmim]Cl was attributed to the presence of carboxylate and hydroxyl groups at the surface of the Cdots. ${ }^{[5]}$ In contrast, CdotsCHI/[Bmim] Cl/[Tmi][Trif] exhibits a zeta potential of $+0.66 \mathrm{mV}$ (Figure $4 \mathrm{a}$ ). While the positive value is associated with the presence of amino groups on the Cdots surface, ${ }^{[24]}$ the fact that the value is close to zero further confirms the aggregation tendency detected by HR-TEM and AFM.

\subsection{Nanofluids Fluorescence Properties}

Up until recently the optical properties of the Cdots have been extensively studied using steady-state and time-resolved fluorescence techniques ${ }^{[25]}$ in order to provide a better knowledge of the fluorescence mechanism of these nanoparticles. However the fluorescence dependence with temperature, that can indeed offer great insight on the subject, has been practically neglected.

We performed an analysis of the fluorescence dependence of the nanofluids with temperature in the $16-50{ }^{\circ} \mathrm{C}$
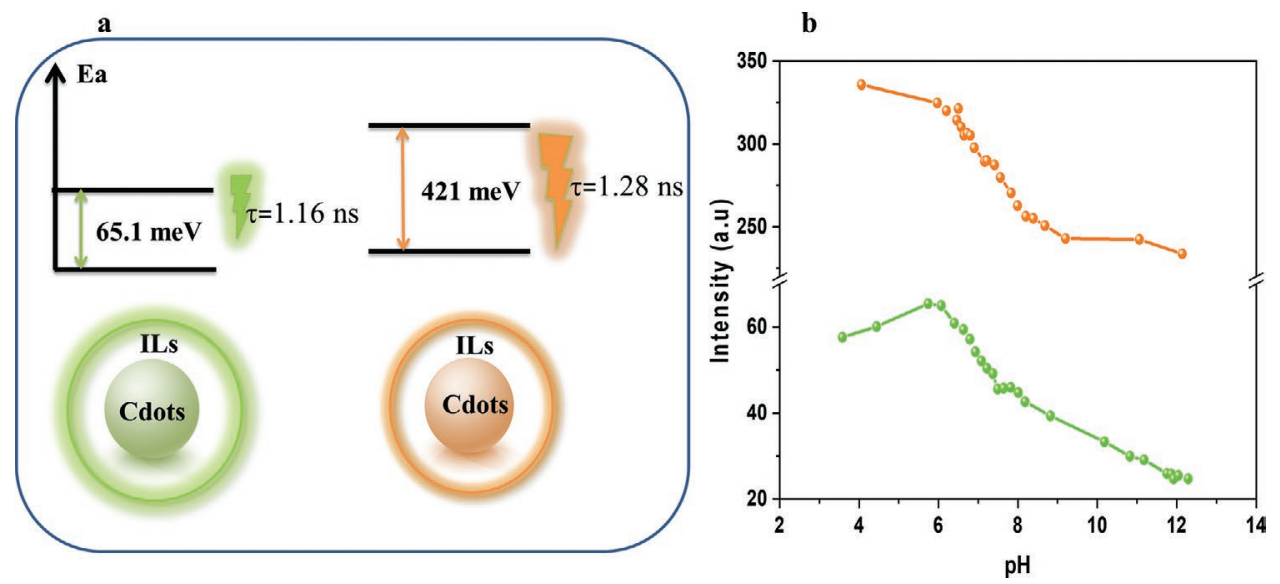

Figure 4. Fluorescence dependence with temperature and $\mathrm{pH}$. a) Schematic representation of the activation energy $(E)$ and fluorescence lifetime $(\tau)$ for Cdots/[Bmim] Cl/[Tmi][Trif] (green) and Cdots CHI/[Bmim] Cl/[Tmi][Trif] (orange). b) Fluorescence intensity profile variation with $\mathrm{pH}$ using the maximum emission wavelength of $500 \mathrm{~nm}$ for both nanofluids aqueous solutions. 
range (see further details in section below). The fluorescence intensity decreased progressively as the temperature increased. This trend can be attributed to thermallyactivated non-radiative trapping. ${ }^{[25]}$ Based on the equation $\left.I(T)=\frac{I_{0}}{1+\left(\tau_{\mathrm{r}} / \tau_{0}\right) \times \exp \left(-E \mathrm{a} / K_{\mathrm{B}} T\right)}\right]$, where $T$ is temperature

(in $\mathrm{K}$ ), Ea is the activation energy, $\tau$ is the fluorescence lifetime, $\eta=\left(1+\tau_{\mathrm{r}} / \tau_{\mathrm{nr}}\right) T^{-1}$ represents the quantum efficiency, and $\mathrm{r}$ and $\mathrm{nr}$ stand for radiative and non-radiative, respectively, it was possible to determine the Ea value for Cdots/[Bmim] Cl/[Tmi] [Trif] and CdotsCHI/[Bmim]Cl/[Tmi][Trif] (65.1 \pm 3 and $421 \pm$ $10 \mathrm{meV}$, respectively) (Figure 4a). The significantly lower Ea value obtained for Cdots/[Bmim]Cl/[Tmi][Trif] with respect to CdotsCHI/[Bmim]Cl/[Tmi][Trif] provides evidence that Cdots/ $[\mathrm{Bmim}] \mathrm{Cl} /[\mathrm{Tmi}][$ Trif] is composed of Cdots with a higher density of defect states. Nonetheless both values are higher than those obtained for bare (non-functionalized) Cdots. ${ }^{[25]}$ This finding is a solid indication that the functionalization in situ of the Cdots with the ILs helped, not only to stabilize the NP surface, but also to decrease the surface defects.

Additionally, due to the ILs chemical properties, we decided to perform an acid-base titration and determine the apparent $\mathrm{p}$ Ka for all the nanofluids. Although these nanofluids show polyelectrolyte behavior, typical in functionalized Cdots, ${ }^{[20]}$ the fluorescence intensity variation can be linearized using the Henderson-Hasselbach equation: $\mathrm{pH}=\mathrm{pKa}+n \log \left[\frac{I_{\max }-I}{I-I_{\min }}\right]$, where $I_{\max }$ and $I_{\min }$ correspond to the maximum and minimum fluorescence intensity of the acid or conjugated base species, respectively, and $I$ is the fluorescence intensity as a function of the $\mathrm{pH}$. The parameter $n$ is used due to the predictable polyelectrolyte behavior, usually greater than unity. ${ }^{[20]}$ The apparent $\mathrm{pKa}$ values obtained for the two nanofluids containing [Tmi][Trif] are similar $(8.2 \pm 0.1$ and $7.4 \pm 0.1$, respectively). These values are relatively close to that described in the literature for aqueous solutions of ILs comprising the [Trif $\left.{ }^{-}\right]$counter ion $(\mathrm{pKa}=7.0),{ }^{[26]}$ but markedly different from that obtained for Cdots/[Bmim]Cl under the same conditions $(3.3 \pm 0.2)$. Additionally, in all the nanofluids aqueous solutions $n>1$, which is an indication of their polyelectrolyte nature. Moreover, in aqueous solutions all the nanofluids presented an acidic nature $(\mathrm{pH}=5.8,3.6,4.1$, for Cdots/[Bmim]Cl, Cdots/[Bmim]Cl/[Tmi][Trif], and CdotsCHI/ $[\mathrm{Bmim}] \mathrm{Cl} /[$ Tmi $][$ Trif], respectively). Interestingly, it was previously reported ${ }^{[27]}$ that the conversion of cellulose, a complex carbohydrate, to reducing sugars, such as hydroxymethylfurfural (a precursor for the Cdots synthesis), is limited by the acidity of the IL. Moreover, Choi et al. ${ }^{[28]}$ proved that the counter ion has to be active enough to allow the reaction completion. This could be the reason why the production of Cdots from chitin using only [Bmim]Cl was not possible. The acidity of this IL may not be enough to promote the cascade reaction starting from the $\mathrm{N}$-glycosidic cleavage between the chitin monomers until the formation of the carbon clusters and the subsequent Cdots.

\subsection{Thermosensitive Behavior of the Nanofluids}

The zeta potential of the Cdots/[Bmim]Cl/[Tmi][Trif] and CdotsCHI/[Bmim]Cl/[Tmi][Trif] nanofluids are markedly dependent on the temperature (Figure 5a, green and orange symbols, respectively). In the case of Cdots/[Bmim]Cl/[Tmi] [Trif] an anomalous behavior was detected between 30 and $35^{\circ} \mathrm{C}$, both in the heating and cooling cycles (Figure 5a, green symbols), which can be associated with the TT transition of [Bmim]Cl. During cooling the zeta potential decreased progressively reaching a final value of $\approx-20 \mathrm{mV}$ at $16{ }^{\circ} \mathrm{C}$ (Figure 5a, green symbols), probably as a consequence of the breakdown of the hydrogen bonded network which very likely promoted the formation of additional negative species. At $16{ }^{\circ} \mathrm{C}$ CdotsCHI/[Bmim]Cl/[Tmi][Trif] exhibited a value of $+0.66 \mathrm{mV}$ that increased to $+7.1 \mathrm{mV}$ at $50{ }^{\circ} \mathrm{C}$ (Figure $5 \mathrm{a}$, orange symbols), indicating that at the latter temperature the NPs were close to aggregation, although their stability dramatically increased with temperature. In this nanofluid the TT transition is not discerned (Figure 5a, green symbols). This observation beautifully corroborates the explanation that the butyl groups of the imidazolium cations are interacting with the surface of the Cdots and are thus less prone to undergoing a conformational change.

The UV/vis absorption profiles of the Cdots/[Bmim]Cl/[Tmi] [Trif] and CdotsCHI/[Bmim]Cl/[Tmi][Trif] nanofluids show a weak UV/vis absorption tail that extends to the vis region of the spectrum (Figure 5b, green and orange symbols, respectively). The maximum peak absorption was upshifted to $280 \mathrm{~nm}$ in the case of Cdots/[Bmim]Cl/[Tmi][Trif], and downshifted to $233 \mathrm{~nm}$ in the case of CdotsCHI/[Bmim] Cl/[Tmi][Trif], with respect to Cdots/[Bmim]Cl $\left(263 \mathrm{~nm}^{[5]}\right)$. This maximum wavelength mismatch reinforces the fact that both Cdots present different groups around their surface, in spite of the fact that the Cdots in the present two nanofluids contain the same ILs. This observation strongly suggests that the difference found can only be due to the different nature of the carbon source used to produce them.

Another feature that is strongly dependent, not only on the surface groups and defects that arise from the synthesis method, but also on the starting materials, is fluorescence. Up until now the intrinsic behavior of the Cdots fluorescence variation with temperature has not yet been fully explored in the literature. However, its knowledge can allow us to get a better insight into the fluorescence mechanism that lies beneath these NPs and consequently the nanofluids they are a part of. Figure $5 c$ shows a consistent fluorescence intensity decrease with increasing temperature for both nanofluids. A closer look reveals, however, that, in spite of the fact that both nanofluids display a similar tendency, their particular behavior differs. Indeed, and even though the global fluorescence intensity decrease is essentially the same within the 16 and $50{ }^{\circ} \mathrm{C}$ interval (15\% for Cdots/[Bmim]Cl/[Tmi][Trif] and 13\% for CdotsCHI/[Bmim]Cl/[Tmi][Trif]), in the case of Cdots/[Bmim] $\mathrm{Cl} /\left[\right.$ Tmi][Trif] between 32 and $36{ }^{\circ} \mathrm{C}$ the fluorescence intensity reaches a plateau and then returns to the decreasing trend. This temperature range coincides with that of the TT transition of $[\mathrm{Bmim}] \mathrm{Cl}^{[29]}$ due to the trans-trans/trans-gauche conformational change of the butyl group. Therefore, although both nanofluids include $[\mathrm{Bmim}] \mathrm{Cl}$, its TT transition is more pronounced in Cdots/[Bmim] Cl/[Tmi][Trif]. It is noteworthy to recall again two facts at this stage: 1 ) While Cdots/[Bmim]Cl/[Tmi][Trif] is composed of a homogenous size dispersion of Cdots, CdotsCHI/ $[$ Bmim] $\mathrm{Cl} /[$ Tmi] $]$ Trif] contains two sized populations that may mask the temperature effect associated with the butyl group 

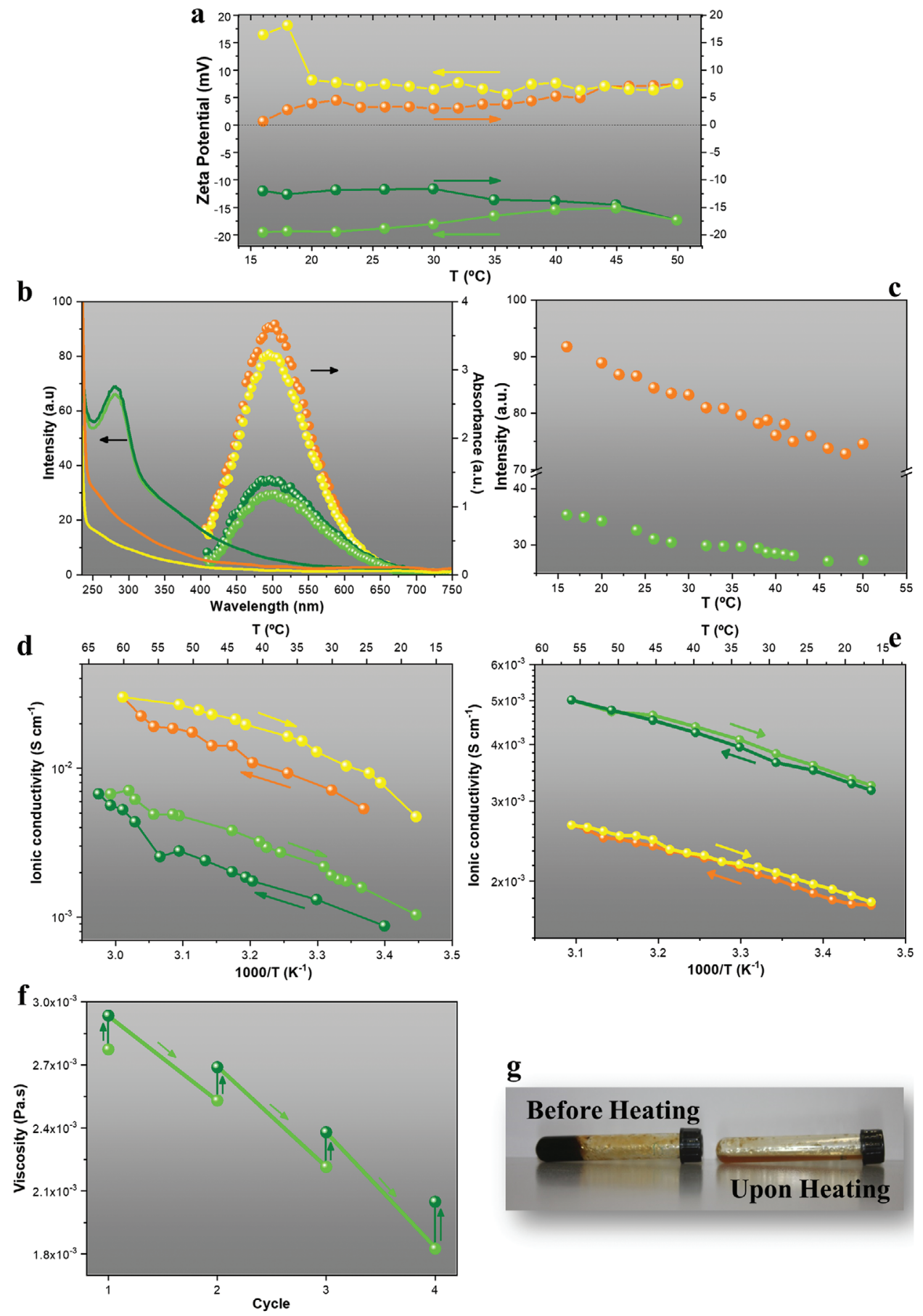

Figure 5. Thermosensitivity of the Cdots/[Bmim] Cl/[Tmi][Trif] (light green and dark green) and $\mathrm{Cdots} \mathrm{CHI} /[\mathrm{Bmim}] \mathrm{Cl} /[\mathrm{Tmi}][$ Trif] (yellow and orange) nanofluids. a) Zeta potential in the first heating (dark color symbols)/cooling (light color symbols) cycle. b) Fluorescence intensity (symbols) and absorbance (lines) at $16{ }^{\circ} \mathrm{C}$ (light color symbols) and $36^{\circ} \mathrm{C}$ (dark color symbols). c) Fluorescence intensity acquired at the maximum emission wavelength $(503 \mathrm{~nm}$ ) using a $400 \mathrm{~nm}$ excitation wavelength. d) lonic conductivity in the bulk state during the first heating (dark color symbols)/cooling (light color symbols) cycle. e) lonic conductivity of the $95 \% \mathrm{w} / \mathrm{w}$ aqueous solutions during the first heating (dark color symbols)/cooling (light color symbols) cycle. f) Dynamic viscosity at $27^{\circ} \mathrm{C}$ in four consecutive heating (dark color symbols)/cooling cycles (light color symbols). g) Appearance of Cdots/[Bmim] $\mathrm{Cl} /[$ Tmi] $]$ Trif] before heating and upon the first heating/cooling cycle.

of [Bmim]Cl; 2) We noted above that in Cdots/[Bmim]Cl/[Tmi] [Trif] and CdotsCHI/[Bmim]Cl/[Tmi][Trif] the imidazolium cations interact with the functional groups of the surface of the Cdots via the ring $\mathrm{N}$ atoms and the butyl groups, respectively.
This means that in the latter case the conformational transition will be very probably hindered due to steric effects. Previous studies ${ }^{[29-32]}$ using semiconductor quantum dots (QDs) pointed out that the fluorescence full width half maximum 
(FWHM) is a temperature-independent parameter. When variations occur, they are due to the presence of exciton-phonon interactions and ionized impurity scattering. In the case of the Cdots/[Bmim]Cl/[Tmi][Trif] and CdotsChi/[Bmim]Cl/[Tmi] [Trilf] nanofluids the FWHM decreased $14 \%$ and $10 \%$, respectively, in the $16-50{ }^{\circ} \mathrm{C}$ interval. This small variation is definitely a particular feature of the nanofluid. In addition, the data of Figure $5 c$ provide rich information on surface defects. Both nanofluids present a Stokes shift of $103 \mathrm{~nm}$ (acquired at the maximum emission wavelength [503 nm] using a $400 \mathrm{~nm}$ excitation wavelength), which is consistent with the occurrence of NPs, particularly Cdots. ${ }^{[13,20]}$ Moreover, the nanofluid emission band has a FWHM > $100 \mathrm{~nm}$, even at low temperatures, which can be attributed to strong electron-electron interactions. This is actually quite interesting, since this is a behavior previously reported for the extensively studied metallic-based QDs. Considering that Cdots have usually a similar fluorescence behavior, we are led to suggest that the $\pi$-electrons in the Cdots of the nanofluid act in the same way free-electrons do in the metallic nanoclusters.

The Cdots/[Bmim] Cl nanofluid exhibited high ionic conductivity between 20 and $65^{\circ} \mathrm{C},{ }^{[5]}$ as a result of proton transport via Gotthus hopping and/or vehicular mechanisms. Figure 5d,e clearly demonstrates that the introduction of a second IL around the Cdots (i.e., [Tmi][Trif]) was, as envisaged, clearly advantageous. Comparison of the ionic conductivity of Cdots/[Bmim] $\mathrm{Cl}^{[5]}$ and Cdots/[Bmim]Cl/[Tmi][Trif] (Figure 5d, green symbols) nanofluids over the same temperature interval allows inferring that the inclusion of [Tmi][Trif] exerted a remarkable increase from $0.046^{[5]}$ to $1 \mathrm{mS} \mathrm{cm}^{-1}$ at $25^{\circ} \mathrm{C}$, and from $2.19^{[5]}$ to $4.81 \mathrm{mS} \mathrm{cm}^{-1}$ at $50^{\circ} \mathrm{C}$. For CdotsCHI/[Bmim]Cl/[Tmi][Trif], the enhancement of the ionic conductivity induced by [Tmi] [Trif] was even higher. For instance, comparison of the values measured at $50{ }^{\circ} \mathrm{C}$ for the Cdots/[Bmim] Cl and CdotsCHI/ $[\mathrm{Bmim}] \mathrm{Cl} /[$ Tmi] $]$ Trif $]$ allows concluding that the increase corresponded to an order of magnitude (from $2.19^{[5]}$ to $26.8 \mathrm{mS} \mathrm{cm}^{-1}$ (Figure 5e, red symbols)). Taking into account that both nanofluids were functionalized with the same ILs, we feel tempted to suggest that the higher conductivity of CdotsCHI/[Bmim] Cl/ [Tmi][Trif] is related with the relative orientation of $\left[\mathrm{Bmim}^{+}\right]$ and $\left[\mathrm{Tmi}^{+}\right]$cations around the surface of the Cdots. As mentioned above, in CdotsCHI/[Bmim]Cl/[Tmi][Trif] the imidazolium cations interact with the surface of the NPs via the alkyl groups, meaning that the $\mathrm{N}$ atoms of the imidazolium rings are directed outwards. Consequently these Lewis bases, which have excellent proton acceptor ability, may participate actively in proton transport. Another interesting conclusion that can be drawn from Figure 5d,e is that for both nanofluids the ionic conductivity increased regularly with successive heating/ cooling cycles as a result of non-reversible hysteresis, and the initial value is never recovered (Figure S4, Supporting Information). This effect was also discerned in Cdots/[Bmim] $\mathrm{Cl}^{\left[{ }^{[5]}\right.}$ This continuous improvement of the ionic conductivity may be interpreted as a fingerprint of complexity, revealing a selforganization process. The latter is intimately associated with a network of hydrogen bonds which are disrupted upon heating, but are never re-established exactly upon cooling. The analysis of the temperature dependence of the ionic conductivity of the $95 \% \mathrm{w} / \mathrm{w}$ nanofluid aqueous solutions shown in Figure $5 \mathrm{e}$ is also extremely interesting. We reported that a distinct feature of the Cdots/[Bmim]Cl nanofluid was the fact that its ionic conductivity was higher than that of a $95 \% \mathrm{w} / \mathrm{w}$ nanofluid aqueous solution. ${ }^{[5]}$ It may be inferred from Figure $5 \mathrm{~d}$ that, while the same comment is valid for CdotsCHI/[Bmim] Cl/ [Tmi][Trif], a completely different behavior is displayed by the $95 \% \mathrm{w} / \mathrm{w}$ nanofluid aqueous solution of Cdots/[Bmim] Cl/[Tmi] [Trif] which exhibits ionic conductivity values which do not differ much from those of the corresponding bulk nanofluids below $55^{\circ} \mathrm{C}$. We note that in the case of the $95 \% \mathrm{w} / \mathrm{w}$ nanofluid aqueous solutions, the hysteresis is considerably less important than in the case of the bulk nanofluids.

Typically, a temperature increase is followed by an increase in the molecules mobility and, consequently, in the molecular interchange. When this phenomenon takes place in the liquid state it is also necessary to consider the additional attractive, cohesive forces established between the liquid molecules. In this sense it may be stated that both molecular interchange and cohesion forces contribute to the liquid viscosity. Upon increasing the temperature, there is a decrease in the attractive binding energy and, therefore, in the liquid viscosity. In the case of the new Cdots/[Bmim]Cl/[Tmi][Trif] nanofluid, the dynamic viscosity decreased progressively as the temperature was increased during consecutive heating/cooling cycles (Figure 5f), a result that is in perfect agreement with the behavior found for Cdots/[Bmim]Cl. ${ }^{[5]}$ This behavior was reproducible over at least five cycles. When the solution was left in rest for $48 \mathrm{~h}$ after being heated, the initial viscosity was not recovered, a behavior typical of non-Newtonian flow. ${ }^{[23,33]}$ To test if the viscosity effect observed was solely triggered by temperature, a nanofluid sample just prepared was left at room temperature for more than two weeks. After this time, no significant viscosity change was observed. The latter result is quite interesting, since it provides further evidence that the nanofluids characteristics remain constant overtime unless a significant energy input alters it. The fact that the initial value was never attained after consecutive heating/cooling cycles, was also observed during the ionic conductivity measurements, as noted above, and during the fluorescence analysis (not shown).

In short, the above data demonstrate that the intrinsic characteristics of the Cdots/[Bmim]Cl/[Tmi][Trif] nanofluid are improved with successive heating/cooling cycles. This is a quite relevant feature since in real-life application as coating materials they will be indeed naturally exposed to several heating/ cooling cycles.

Nanofluids present several advantages over the base fluid. An aspect that has been gathering much attention in the energy area is their thermal sensitivity. ${ }^{[2,23,34]}$ To investigate this intrinsic characteristic in the case of the Cdots/[Bmim] Cl/ [Tmi][Trif] and CdotsCHI/[Bmim]Cl/[Tmi][Trif] nanofluids, we analyzed the temperature dependence of the TT effect, the zeta potential, the optical properties, the ionic conductivity, and the dynamic viscosity.

Targeting the development of TTDs, a film of, either Cdots/ [Bmim]Cl/[Tmi][Trif] or CdotsCHI/[Bmim]Cl/[Tmi][Trif], was spread between two plain (Figure S4, Supporting Information) or silver (Ag)-decorated (Figure S5, Supporting Information) glass slides. While the use of the plain glass slides allowed evaluating the intrinsic properties of the films, the use of 


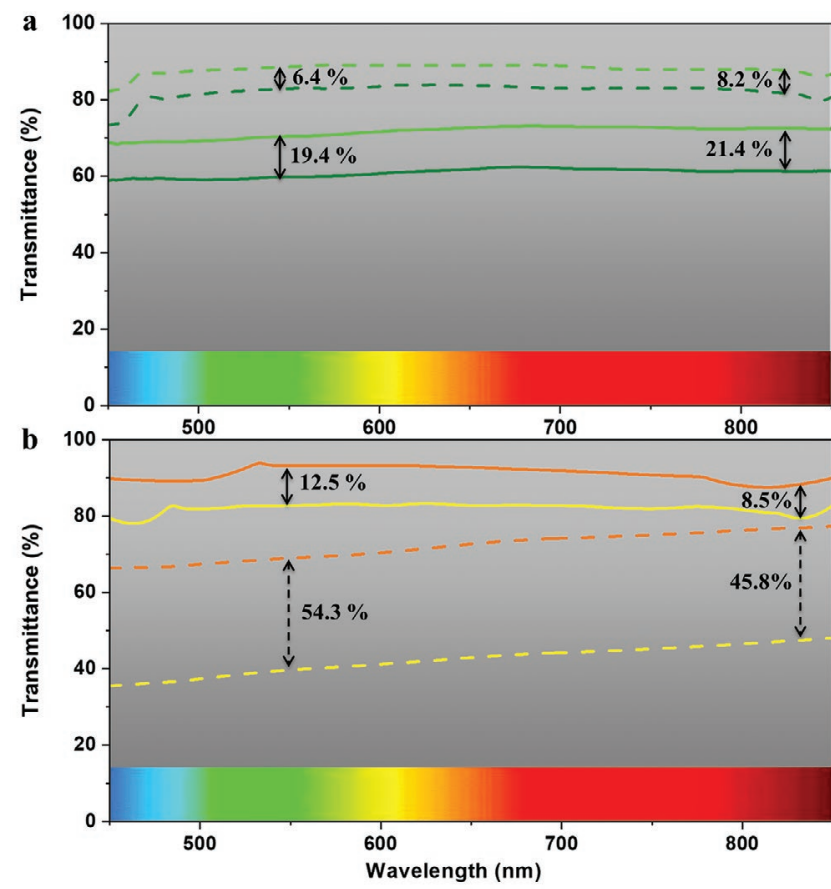

Figure 6. Performance of TTDs. Temperature dependence of the transmission spectra of glass/nanofluid/glass (dotted lines) and glass/ nanofluid/Ag/glass (solid lines) devices incorporating a) Cdots/[Bmim] $\mathrm{Cl} /[$ Tmi][Trif] (green lines) and b) $\mathrm{Cdots} \mathrm{CHI} /[\mathrm{Bmim}] \mathrm{Cl} /[\mathrm{Tmi}][$ Trif] (red) between at $16^{\circ} \mathrm{C}$ (light color) and $50^{\circ} \mathrm{C}$ (dark color).

glass slides incorporating small Ag islands enabled the SPRE (Figure S6, Supporting Information). To guarantee that the films where comparable their thickness was maintained constant $(0.013 \mathrm{~mm})$. The samples employed were the $95 \% \mathrm{w} / \mathrm{w}$ nanofluid aqueous solutions. A marked decrease of the $T$ value resulted upon a temperature increase. Taking as reference the photopic vision wavelength $(550 \mathrm{~nm})$, the response of the two nanofluids in terms of $\Delta T$ to the increase of temperature from 16 to $50{ }^{\circ} \mathrm{C}$ was different: $6.4 \%$ for Cdots/[Bmim]Cl/[Tmi][Trif] (Figure 6a, dotted green lines) and 12.5\% for CdotsCHI/[Bmim] $\mathrm{Cl} /[$ Tmi] [Trif] (Figure 6b (red dotted lines). Moreover, in the case of Cdots/[Bmim]Cl/[Tmi][Trif] the temperature increase was always followed by a decrease of $T$. In contrast, in CdotsCHI/ $[\mathrm{Bmim}] \mathrm{Cl} /[$ Tmi $]\left[\right.$ Trif], there was a marked decrease until $36^{\circ} \mathrm{C}$ and then it seems that the transmittance remained practically unchanged. It is interesting to emphasize that this sensitivity is more marked until this temperature was reached, since this is the thermotropic temperature of [Bmim]Cl. ${ }^{[29]}$ This result seems to prove that the butyl chain of [Bmim]Cl in CdotsCHI/ $[\mathrm{Bmim}] \mathrm{Cl} /[$ Tmi $][$ Trif $]$ has some conformational restrictions that are not present in the other synthetized Cdots. Indeed, as it was discussed above, in these Cdots the alkyl chain should be closer to the NP surface. Since these nanofluids are composed of carbon NPs, it is possible to enhance their heat transfer rates, and absorption/transmission properties by taking advantage of the SPRE.. ${ }^{[35]}$ Figure 6a,b shows that the excitation of the plasmons of the $95 \%$ aqueous solutions of Cdots/[Bmim]Cl/[Tmi] [Trif] and CdotsCHI/[Bmim]Cl/[Tmi][Trif], and their transformation into propagating photons enhanced the $\Delta T$ value to
19.4\% and 54.3\%, respectively. Thus the SRPE was considerably more efficient in the case of the CdotsCHI/[Bmim]Cl/[Tmi][Trif] nanofluid than in Cdots/[Bmim]Cl/[Tmi][Trif]. This is a quite puzzling result. It is accepted that smaller, spherical NPs are more susceptible to the plasmonic effect, and that nanofluids composed of heterogeneous sized NPs are less adequate for this phenomenon. ${ }^{[36,37]}$ In the present case, the opposite seems to happen. Indeed, the Cdots that yielded the best results in terms of thermosensitive behavior (without and with SRPE) are those composed of two main size populations (45 and $170 \mathrm{~nm}$ ), close to aggregation at room temperature. At this stage it is of interest to compare these results with those obtained with Cdots/[Bmim]Cl. ${ }^{[5]}$ The latter nanofluid, which contained monodisperse Cdots with average size of $6.8 \mathrm{~nm},{ }^{[5]}$ led to $\Delta T$ values of $17 \%$ and $61 \%$ without and with SRPE, respectively, therefore pointing out a much better thermo-opto-electronic performance of the TTDs than in the present two cases. Considering that in the $\mathrm{CdotsCHI} /[\mathrm{Bmim}] \mathrm{Cl} /[\mathrm{Tmi}][$ Trif] nanofluid NPs with average sizes similar to those of Cdots/[Bmim $] \mathrm{Cl}$ (of the order of $10 \mathrm{~nm}$ ) were also detected by HR-TEM (not shown), we feel tempted to speculate that the reason for the better performance of $\mathrm{CdotsCHI} /[\mathrm{Bmim}] \mathrm{Cl} /[\mathrm{Tmi}][$ Trif $]$ with respect to Cdots/[Bmim]Cl/[Tmi][Trif] relies on the presence of these $10 \mathrm{~nm}$-sized NPs. In this sense, and assuming that our claim is correct, the fact that the reaction did not go to completion in the case of CdotsCHI/[Bmim]Cl/[Tmi][Trif] was after all beneficial.

\section{Conclusion}

In this work we describe for the first time the synthesis of a Cdots-based nanofluid produced from chitin originating from crab shells using ILs as reaction media and functionalizing molecules. Moreover, we demonstrated that according to the raw material used in the Cdots synthesis (glucose or chitin) it was possible to obtain NPs with different sizes, chemical groups, and surface defects. All these parameters greatly affect all the intrinsic characteristics of the nanofluids, namely, their optoelectronic properties, wettability, thermal sensitivity, among others. According to the results obtained in this work it is possible to conclude that the nanofluid with smaller NPs and higher homogeneity in size was obtained using glucose as starting material. Using chitin requires an adjustment in the reaction conditions in order to obtain a homogenous size distribution. Indeed, since the raw material has a higher complexity, it is necessary to increase the reaction time. Nonetheless, despite the fact that the nanofluid derived from chitin with a reaction time of only $1 \mathrm{~h}$ reaction time had two distinct size populations, it was still possible to observe a higher thermal sensitivity in it optoelectronic properties. Moreover, when we produced a thin film on glass slides, this non-Newtonian nanofluid thermal sensitivity was greatly enhanced by the plasmonic effect provided by small Ag islands immobilized on the glass. This result provided further evidence that the homogeneity, size, and chemical groups of the NPs are of the outmost importance in the development of high-efficient thermal systems. The results obtained in this work provide a deeper understanding of, not only non-Newtonian nanofluids based on Cdots, but 
also point out their potential applications as coating films with improved thermal sensitivity.

\section{Experimental Section}

Materials: Room temperature ionic liquids (ILs), 1-methylimidazole ([Hmim $][\mathrm{Cl}])$, and 1-butyl-3-methylimidazolium chloride ([Bmim] $\left.[\mathrm{Cl}]-\mathrm{IL}_{1}\right)$ were obtained from Merck and Acros Organics, with a purity of $99 \%$ and $98 \%$, respectively, Trifluoromethanesulfonic acid was obtained from Merck with a purity of $99 \%$. Anhydrous Acetone was obtained from Fluka, Chitin (from crab shells) from Sigma-Aldrich, and anhydrous glucose from Fisher Scientific all with analytical grade. p-toluene sulfonic acid was obtained from Alfa-Aeser with $98.5 \%$ purity. All the chemicals were used as received unless otherwise stated. High-purity Milli-Q water (resistivity $18 \mathrm{M} \Omega \mathrm{cm}$, Interlab Sistem Purist UV set) was used in all aqueous solutions.

Synthesis of the Ionic Liquid: 1-Tosylate-3-methyl-imidazolium Triflate $\left[\mathrm{Tmi}^{+}\right]\left[\mathrm{Tfl}^{-}\right]\left(\mathrm{IL} \mathrm{L}_{2}\right)$ : This synthesis was performed in two concerned steps: Step A-Trifluoromethanesulfonic acid was carefully added to anhydrous acetone in 1:12 ratio. Afterward, a solution of 1-methylimidazole in anhydrous acetone with the same molar ratio was added dropwise under constant stirring. A coloration change was observed going from light orange, red up until brown. This coloration remained constant overtime. Step B-The as-prepared solution was added to $10 \mathrm{mmol}$ p-toluene sulfonic acid under constant stirring and using an ice bath. The obtained mixture was heated to $100{ }^{\circ} \mathrm{C}$ and left under stirring overnight. The $\mathrm{IL}_{2}$ was a black viscous liquid. In order to remove the solvent the obtained $\mathrm{IL}_{2}$ was placed in a vacuum system, without further heating. When the volume was constant the solution was loaded into dialysis molecular membrane (molecular mass cutoff: $500-1000 \mathrm{Da}$ ) and dialyzed against ultrapure water for $48 \mathrm{~h}$ in order to remove any unreacted reagents. The obtained solution was then lyophilized for $48 \mathrm{~h}$ and a viscous black liquid was obtained and used without further treatment.

In Situ Synthesis of the Nanofluid Based on Carbon Dots Functionalized with Both lonic Liquids: The single step synthesis of the nanofluid based on Cdots was performed in a round-bottom flask with constant magnetic stirring. Cdots obtained from anhydrous glucose (Cdots/[Bmim] Cl/[Tmi $\left.{ }^{+}\right]$ [Trif-]) and Cdots produced through the decomposition of crab shells Chitin (Cdots $\mathrm{CHI}$ ) were obtained from the addition of $\mathrm{IL}_{1}$ to anhydrous glucose or Chitin in a 1:10 $\mathrm{m} / \mathrm{m}$ ratio under constant magnetic stirring. Afterward, $1 \mathrm{~mL}$ of the pure $\mathrm{IL}_{2}$ was added to the mixture and left to react at $100{ }^{\circ} \mathrm{C}$ in an oil bath for $1 \mathrm{~h}$. The resulting NPs were left to cool at room temperature. After cooling, the resulting Cdots presented a viscous consistency, Cdots/[Bmim $] \mathrm{Cl} /\left[\mathrm{Tmi}^{+}\right]\left[\mathrm{Trif}^{-}\right]$had a brown coloration, and $\mathrm{Cdots} \mathrm{CHI}$ had an orange color. They were transferred into a dialysis cellulose tubing membrane with a molecular weight cutoff of $500 \mathrm{Da}$ and were dialyzed against ultrapure water for at least $72 \mathrm{~h}$. The resulting solution was centrifuged for $10 \mathrm{~min}$ at $5000 \mathrm{rpm}$ and the supernatant was extracted and centrifuged again. This procedure was repeated three times, until no solid could be separated from the solution. The resulting solutions were lyophilized until no water was found, which was roughly three days. The purified Cdots had a viscous nanofluidic consistency at room temperature. Upon lyophilization the nanofluid could easily be re-dispersed in water and other solvents whenever necessary.

Characterizations: The $\mathrm{H}^{\top} \mathrm{NMR}$ spectrum was recorded at room temperature on a Bruker Avance II+ $400(400 \mathrm{MHz})$ in C.A.C.T.I. Universidade de Vigo. Deuterated DMSO was used as solvent. (Figure S7, Supporting Information) High-resolution transmission electron microscopy images were recorded on a JEOL JEM 2100 $(200 \mathrm{kV})$ with UC-A on holey 400 mesh Cu grids (Ted Pella ref. 01824). The preparation of the samples started by dispersing them in ethanol and when a homogeneous solution was obtained, the nanofluids were placed into the analyzing grid by drop-casting, followed by drying at room temperature. TGA was performed using a TG209 F3 Tarsus thermogravimetric analyzer (Nietzsch Instruments) under an $\mathrm{N}_{2}$ atmosphere with a heating rate of $10{ }^{\circ} \mathrm{C} \mathrm{min}^{-1}$. UV-vis spectra were obtained using a UV-vis spectrophotometer (U-4100) and a
$10 \mathrm{~mm}$ path quartz cuvette. The dynamic viscosity measurements were performed on a rheometer (Brookfield DV-II) equipped with a cone-plate system (SSA spindle 21). Conductivity measurements were performed on WPA CM35 analog conductivity meter with a homemade probe with a constant value of $0.5468 \mathrm{~cm}^{-1}$. These two last parameters, viscosity and conductivity were acquired as a function of temperature $\left(5\right.$ and $\left.60^{\circ} \mathrm{C}\right)$, as such, during measurements the sample holder was kept inside a Julabo F25 thermostatic bath. The nanofluids zeta potential was measured with a Zeta PALS potential analyzer (Brookhaven, USA) with parallel-plate platinum black electrodes spaced $5 \mathrm{~mm}$ apart and a $10 \mathrm{~mm}$ path length rectangular disposable polystyrene cell. All the samples were measured using a sinusoidal voltage of $80 \mathrm{~V}$ with a frequency of $3 \mathrm{~Hz}$. For each sample, in total ten values were automatically given by the instrument, which were then averaged to give the final value and the standard deviation. The AFM measurements were performed in tapping mode using an AFM CSI Nano-Observer equipment (Scientec) and a super sharp Si HQ:NSC19/ FORTA probe that had a frequency resonance of $60 \mathrm{kHz}$ and a spring constant of $0.3 \mathrm{~N} \mathrm{~m}^{-1}$. The data collected were analyzed using the Gwyddion 2.52 software and the quality of the images were improved using the flattening and elimination of line noise tools. The static contact angle measurements were performed on a Krüss DSA25S drop shape analyzer controlled by the software ADVANCE in a temperaturecontrolled chamber at $25 \pm 1{ }^{\circ} \mathrm{C}$. The volume of the liquid droplets used was $5 \mu \mathrm{L}$. The contact angles were measured using digital images acquired by a video camera and processed by the YoungLaplace fitting. On each sample at least five independent droplets were analyzed and on each droplet five contact angles were acquired within 1-3 min range. The results here presented corresponded to an average value for all these described measurements. The error analysis of the data was implemented by arithmetic mean of the root mean square error.

\section{Supporting Information}

Supporting Information is available from the Wiley Online Library or from the author.

\section{Acknowledgements}

This research was funded by National Funds by Foundation for Science and Technology (FCT) and by FEDER funds through POCI-COMPETE 2020-Operational Programme Competitiveness and Internationalization in Axis I-Strengthening Research, Technological Development and Innovation (UID/QUI/00616/2013, UID/QUI/50006/2019, UID/ Multi/00709/2013, UID/QUI/00313/2019, UID/CTM/50025, POCI-010145-FEDER-007491, POCI-01-0145-FEDER-007688, UID/CTM/50025, POCI-01-0145-FEDER-016884, PTDC/CTM-NAN/0956/2014, SAICT/ PAC/0032/2015, POCI-01-0145-FEDER-016422, and NORTE-01-0145FEDER-030858). R.F.P.P. acknowledges FCT-MCTES for SFRH/ BPD/87759/2012 grant. E. Pereira (FCUP, Porto) is acknowledged for her assistance.

\section{Conflict of Interest}

The authors declare no conflict of interest.

\section{Author Contributions}

The manuscript was written through contributions of all authors. All authors have given approval to the final version of the manuscript. 


\section{Keywords}

carbon dots, ionic liquids, nanofluids, plasmonic effect, self-improving ionic conductivity

Received: December 30, 2019

Revised: March 31, 2020

Published online: May 28, 2020

[1] S. K. Das, S. U. Choi, W. Yu, T. Pradeep, Nanofluids: Science and Technology, John Wiley \& Sons, Inc., Hoboken, NJ 2007.

[2] Heat Transfer Enhancement and Thermal Management for Space Applications Employing Femtosecond Laser Processed Metallic Surfaces with Micro/Nanostructures, https://www.nasa.gov/content/heat-transfer-enhancement-and-thermal-management-forspace-applications-employing/\#backtoTop (accessed: June 2019).

[3] S. K. Das, S. U. S. Choi, Adv. Heat Transfer 2009, 41, 81.

[4] S. A. Khan, M. A. Atieh, M. Koç, Energies 2018, 11, 3189.

[5] H. M. R. Gonçalves, R. F. P. Pereira, E. Lepleux, T. Carlier, L. Pacheco, S. Pereira, A. J. M. Valente, E. Fortunato, A. J. Duarte, V. de Zea Bermudez, Adv. Sustainable Syst. 2019, 3, 1900047.

[6] G. E. LeCroy, S. T. Yang, F. Yang, Y. Liu, K. A. S. Fernando, C. E. Bunker, Y. Hu, P. G. Luo, Y. P. Sun, Coord. Chem. Rev. 2016, 320-321, 66

[7] J. Zhang, S. H. Yu, Mater. Today 2016, 19, 382.

[8] M. Havrdova, K. Hola, J. Skopalik, K. Tomankova, M. Petr, K. Cepe, K. Polakova, J. Tucek, A. B. Bourlinos, R. Zboril, Carbon 2016, 99, 238.

[9] V. Y. Rudyak, Adv. Nanopart. 2013, 02, 266.

[10] A. Pal, N. Natu, A. Ahmada, A. Chattopadhyay, J. Mater. Chem. A 2018, 6, 4111

[11] C. Wang, C. Wang, P. Xu, A. Li, Y. Chen, K. Zhuo, J. Mater. Sci. 2016 51,861

[12] X. Pan, Y. Zhang, X. Sun, W. Pan, G. Yu, S. Si, J. Wang, Mater. Res. Express 2018, 5, 045603.

[13] J. C. G. Esteves da Silva, H. M. R. Gonçalves, TrAC, Trends Anal. Chem. 2011, 30, 1327.

[14] T. Chen, Q. Yang, P. Xu, L. Sun, D. Sun, K. Zhuo, ACS Omega 2017, 2, 5251 .
[15] H. Li, L. Chen, H. Wu, H. He, Y. Jin, Langmuir 2014, 30, 15016.

[16] J. Briscoe, A. Marinovic, M. Sevilla, S. Dunn, M. Titirici, Angew. Chem., Int. Ed. 2015, 54, 4463.

[17] X. Sun, Q. Zhang, K. Yin, S. Zhou, H. Li, Chem. Commun. 2016, 52, 12024

[18] X. Liu, J. Pang, F. Xu, X. Zhang, Sci. Rep. 2016, 6, 31100.

[19] O. Ryosuke, H. Satoshi, S. Satyen, K. Akiko, H. Hiro-o, Chem. Lett. 2003, 32, 948.

[20] H. Gonçalves, P. A. S. Jorge, J. R. A. Fernandes, J. C. G. E. da Silva, Sens. Actuators, B 2010, 145, 702.

[21] M. Q. Tran, Y. Yamaguchi, K. Yamatoya, S. Horikoshi, K. Nakata, Inorg. Chem. Commun. 2018, 96, 1

[22] M. Ahmadi, G. Willing, Int. J. Heat Mass Transfer. 2018, 118, 40.

[23] B. A. K. Naik, A. V. Vinod, Exp. Therm. Fluid Sci. 2018, 90, 132.

[24] H. Yang, Y. Liu, Z. Guo, B. Lei, J. Zhuang, X. Zhang, Z. Liu, C. Hu, Nat. Commun. 2019, 10, 1789

[25] P. Yu, X. Wen, Y. R. Toh, J. Tang, J. Phys. Chem. C 2012, 116, 25552.

[26] T. L. Greaves, C. J. Drummond, Chem. Rev. 2008, 108, 206.

[27] S. Kudo, Z. Zhou, K. Yamasaki, K. Norinaga, J. Hayashi, Catalysts 2013, 3, 757.

[28] J. S. Choi, F. S. H. Simanjuntaka, J. Y. Oh, K. I. Lee, S. D. Lee, M. Cheong, H. S. Kim, H. Lee, J. Catal. 2013, 297, 248.

[29] A. Efimova, G. Hubrig, P. Schmidt, Thermochim. Acta 2013, 573, 162.

[30] P. Yu, X. Wen, Y. R. Toh, J. Tang, J. Phys. Chem. C 2012, 116, 25552.

[31] A. Al Salman, A. Tortschanoff, M. Mohamed, D. Tonti, F. van Mourik, M. Chergui, Appl. Phys. Lett. 2007, 90, 093104.

[32] P. Jing, J. Zheng, M. Ikezawa, X. Liu, S. Lv, X. Kong, J. Zhao, Y. Masumoto, J. Phys. Chem. C 2009, 113, 13545.

[33] Y. Yang, E. A. Grulke, Z. G. Zhang, G. Wu, J. Appl. Phys. 2006, 99, 114307.

[34] V. Y. Rudyak, Adv. Nanopart. 2013, 2, 266.

[35] Y. Zhang, H. Gonçalves, J. C. G. E. Silva, C. D. Geddes, Chem. Commun. 2011, 47, 5313.

[36] A. Agrawal, I. Kriegel, D. J. Milliron, J. Phys. Chem. C 2015, 119, 6227.

[37] K. Aslan, Z. Leonenko, J. R. Lakowicz, C. D. Geddes, J. Fluoresc. $2005,15,643$ 